\title{
Familia humana y fraternidad (política) en la Declaración Universal de Derechos de 1948
}

\author{
Human family and political fraternity in the Universal \\ Declaration of Rights of 1948
}

MARÍA JULIA BERTOMEU*

\begin{abstract}
Resumen: La Declaración de 1948 recuperó ideas filosófico-políticas básicas y fundamentales que habían sido ignoradas en la historia política real, o negadas iusfilosóficamente en la academia con el auge del utilitarismo moral y positivismo jurídico. El primer considerando del preámbulo de la Declaración de 1948 afirma enfáticamente, por ejemplo: que la libertad ha de ser universalizada a "todos" y es inalienable, que la "igualdad" bien entendida dimana de la libertad bien entendida, y que la humanidad no está dividida en razas, etnias, géneros, clases o culturas, sino que forma un todo indivisible al que llama "la familia humana". Y ya en el Artículo 1) de esa misma Declaración se incorpora la "fraternidad", no como un adorno retórico o como un encomiable sentimiento social o -menos- como una consigna culturalmente particularista o arbitraria, sino como un deber exigible de manera universal: "Todos los seres humanos (...) deben comportarse fraternalmente los unos con los otros". Así pues, reflexionar políticamente hoy sobre la fraternidad va de la mano de una reflexión sobre el significado profundo de los derechos humanos fundamentales.
\end{abstract}

Palabras clave: fraternidad, derechos humanos, libertad, familia humana, derechos inalienables.

\begin{abstract}
The 1948 Universal Declaration of Human Rights revived basic and fundamental politico-philosophical ideas that had been ignored in actual politics up to that point and denied by academics as a result of the rise of moral utilitarianism and legal positivism. The first part of the Preamble to the 1948 Declaration emphatically states, for example, that freedom must be universalized to "all" and is inalienable; that "equality" properly understood derives from freedom properly understood; and that humanity is not divided into races, ethnicities, genders, classes or cultures, but forms an indivisible whole which it calls "the human family". Moreover, Article 1 of the Declaration incorporates the notion of "fraternity", not as a rhetorical flourish or as a commendable social feeling - much less as a culturally particularistic or arbitrary slogan - but as a universally valid duty: "All human beings [...] should act fraternally towards one another". Thus, thinking about fraternity from a political perspective today goes hand in hand with thinking about the deeper meaning of fundamental human rights.
\end{abstract}

Key words: fraternity, human rights, liberty, human family, inalienable rights.

Recibido: 10/06/2018. Aceptado: 09/07/2018.

* Universidad Nacional de La Plata, Argentina. Contacto: mjbertomeu@gmail.com 


\section{Introducción}

La idea política de la fraternidad ingresó en la historia de la mano de la Revolución Francesa, particularmente después de 1792, es decir, de la mano de la I República revolucionaria francesa y su nueva Declaración de Derechos Humanos y Ciudadanos y de la República fraternal francesa de 1848. Como los Derechos Humanos, la fraternidad desapareció del vocabulario constitucional tras el golpe de Estado de Termidor (1794), y esa desaparición duró cerca de siglo y medio: los derechos humanos -identificados durante todo el siglo XIX con el Terror, con la República y con la Democracia, términos prácticamente coextensivos para los conservadores y los liberales europeos decimonónicos- no reaparecieron sino tras la derrota política y militar del nazifascismo con la solemne Declaración Universal de Derechos Humanos por las Naciones Unidas en 1948. La Declaración de 1948 recuperó ideas filosófico-políticas básicas y fundamentales que habían sido ignoradas -en la historia política real—por las Constituciones monárquico-liberales del XIX o negadas iusfilosóficamente - en la academia - con el auge del utilitarismo moral y el positivismo jurídico.

El primer considerando del Preámbulo de la Declaración de 1948 afirma enfáticamente, por ejemplo: que la libertad ha de ser universalizada a "todos" y es inalienable, que la "igualdad" bien entendida dimana de la libertad bien entendida, y que la humanidad no está dividida en razas, etnias, géneros, clases o culturas, sino que forma un todo indivisible al que llama "la familia humana". Y ya en el Artículo 1) de esa misma Declaración se incorpora a la "fraternidad", no como un adorno retórico o como un encomiable sentimiento social o - menos - como una consigna culturalmente particularista o arbitraria, sino como un deber exigible de manera universal: "Todos los seres humanos (...) deben comportarse fraternalmente los unos con los otros".

El retorno de las grandes ideas iusfilosóficas revolucionarias vino de la mano de la fuerza de los hechos (y de las armas) y de la experiencia histórico-política real. La academia tenía sus propias inercias: el gran Hans Kelsen, campeón del positivismo jurídico democrático tuvo que conformarse con unas "observaciones metodológicas" muy críticas de la Declaración; otros, como Hannah Arendt, la atacaron frontalmente. No es injusto decir que el utilitarismo y el positivismo jurídico siguieron en buena medida dominando en la vida académica como si nada hubiera pasado, al menos hasta los años 60 del siglo XX en que el utilitarismo como doctrina filosófico-política sucumbió como doctrina académicamente dominante en parte gracias a la batalladora crítica de John Rawls.

El gran prestigio político e ideológico de la Declaración de 1948 no puede engañar sobre el hecho de que nunca contó con simpatías unánimes entre académicos. Tampoco hoy. Tras la derrota del utilitarismo y del iuspositivismo en los años 70, volvieron a proliferar, desde el lado de la derecha académica, teorías filosóficas que, como la Robert Nozick, tratan de poner en duda la naturaleza inalienable de la libertad, o doctrinas que, inspiradas en la reviviscencia de la teoría económica neoclásica, buscan socavar ideológicamente las bases intelectuales de los derechos sociales y económicos. Y, desde una pretendida izquierda académica, han vuelto a proliferar ideas tendentes a negar la unidad básica de la humanidad, y un nuevo relativismo filosófico fundado en la división de la humanidad en "culturas" pretendidamente encapsuladas, en "etnias" o en "géneros". Así pues, deliberar políticamente hoy sobre la 
fraternidad, va de la mano de una reflexión sobre el significado profundo de los derechos humanos fundamentales y sobre los argumentos de sus enemigos filosóficos declarados.

El significado inequívocamente liberador de las clases "domésticas" y, en general, subalternas, vulneradas y vulnerables que tuvo la divisa de la fraternidad revolucionaria cayó en el olvido después de 1848 europeo, aunque cien años más tarde la recuperaba la gran Declaración de Derechos de 1948 en su artículo primero: "Todos los seres humanos nacen libres e iguales en dignidad y derechos y, dotados como están de razón y conciencia, deben comportarse fraternalmente los unos con los otros." Nótese que el comportamiento fraternal va de la mano de la libertad, igualdad, dignidad y derechos.

La nueva Declaración de mediados del siglo XX está desprendida de su forma iusnaturalista, pero no del núcleo republicano de su concepción de la ley y del derecho como constitutivos de la libertad civil y política: hay derechos que son constitutivos y, por tanto, inalienables, y el derecho internacional debe velar por su cumplimiento. Comprender este núcleo republicano es fundamental para entender el significado de la fraternidad en el mundo filosófico-político moderno. Pues bien, afirmar que la Declaración de 1948 tiene un núcleo republicano básico que permite pensar en un concepto de fraternidad ligado al reconocimiento de derechos, supone aclarar algunos puntos constitutivos de tal tradición, por ejemplo:

1) que la libertad es inalienable $y$

2) que los derechos inalienables deben ser universalizados a todos los miembros de la familia humana. Veamos.

\section{La libertad es inalienable}

Declarar que la libertad es un derecho igual e inalienable de todos los seres humanos trae consigo claros efectos jurídicos. Implica, en primer lugar, que siendo la libertad inalienable, el ordenamiento jurídico (nacional e internacional) debe prohibir algunos tipos de contratos -aparentemente voluntarios- en los que las partes enajenan su libertad, cosa que tiene derivaciones jurídicas en el derecho privado y público, nacional e internacional, y, además, que libertad e igualdad no son dos principios normativos en pugna sino que están intrínsecamente conectados.

Si bien la Declaración no se detiene a profundizar expresamente las secuelas jurídicas de la inalienabilidad de los derechos iguales de todos, alegar que ciertos derechos son inalienables e iguales es toda una manifestación de principios y de compromisos con una tradición histórica de larga data.

Con demasiada prisa y bastante frecuencia se tiende a interpretar el debate intra-comisión como un enfrentamiento entre liberales y comunitarios o entre países hegemónicos y periféricos, siendo aquellos los impulsores de los derechos subjetivos (egoístas) y éstos de comunidades y estados soberanos que sólo reconocen derechos colectivos. El debate fue intenso y es bien cierto que algunos de los representantes estaban en las antípodas, el documento final mostró que también otra tradición estuvo presente: la de los derechos constitutivos inalienables, la de la libertad inseparable de la igualdad de derechos, la de la incompatibilidad de la libertad con el temor y la miseria. 
Si la libertad es inalienable, entonces es un derecho "constitutivo", no puedo venderlo ni tampoco regalarlo ni tan siquiera con un supuesto consentimiento. Los derechos instrumentales son enajenables, adquiridos y regulados por el derecho público y privado positivo, pero el derecho público nos impide vender nuestra ciudadanía, no puedo vender mi derecho a la vida y ni tan siquiera partes de mi cuerpo, no me está permitido celebrar contratos privados de esclavitud voluntaria, ni tampoco están permitidos los contratos laborales privados indeterminados en cuanto al tiempo y trabajo (locatio conductio operarum). Todo esto muestra que las raíces del derecho público moderno son republicanas, pues poco o nada tendría para decir un liberalismo partidario de una concepción de libertad como no interferencia (actual y real) ante aquellas decisiones privadas de los individuos que no afectan a terceros. La importancia de este enunciado no puede ser mayor, pues si el derecho público con que hoy contamos no fuese de molde republicano, con origen en el derecho romano (en el que, obvio es decirlo, eran nulos de pleno derecho los contratos voluntarios de esclavitud), estarían permitidos los contratos voluntarios de esclavitud, o la venta del voto y la ciudadanía, y por supuesto no lo están. El libertario Richard Nozick honestamente advirtió -hace ya más de 30 años-, que un liberal consecuente debería aceptar como legalmente permitidos los contratos de alienación de la libertad política con una asociación dominante protectora que asumiera el papel de soberano o, en paralelo con este razonamiento de derecho público aplicado al derecho privado, que un individuo firmara un contrato de esclavitud.

La concepción de la inalienabilidad de la libertad de molde republicano tuvo sus partidarios y disidentes. La compartieron Vitoria, Las Casas, Locke, Kant y Marx, y mucho antes Aristóteles. Lo dejó dicho Vitoria: "la libertad no puede intercambiarse por todo el oro del mundo"; y también Locke: “...dado que los hombres no tienen el poder sobre su propia vida, tampoco pueden por medio de contrato o de su propio consentimiento convertirse en el esclavo, ni tampoco ponerse bajo el poder absoluto y arbitrario de otro que pueda quitarle la vida a placer. Y puesto que no tiene poder absoluto sobre sí mismo, tampoco puede concedérselo a otro “ (Locke, 1998, 23), y por supuesto Kant: "un hombre puede ser su propio señor (sui iuris) pero no propietario de sí mismo (sui dominus), esto es, no puede disponer de sí a su antojo" (Kant, 1989, 270). ${ }^{1}$

La antigua teoría de la inalienabilidad de los derechos constitutivos, como toda teoría, tiene consecuencias distintas cuando los hechos y circunstancias históricas cambian, incluso algunas de ellas insospechadas. Pero su médula normativa permanece a pesar de los mudanzas históricas: la inalienabilidad de la libertad personal y política -la soberanía y la autopropiedad bien entendidas- no es compatible con un concepto negativo de libertad como mera ausencia de interferencia real (aplicada a sujetos que prestan su consentimiento a un pacto de sumisión o de alienación de la propia libertad personal a cambio de un salario o de lo que fuere), si la libertad es inalienable entonces se requieren claras y decididas intervenciones de los estados y de los organismos internacionales con capacidad de hacer cumplir

1 Otros muchos, como Luis de Molina, Francisco Suarez, Grotius, Pufendorf y Hobbes, se alinearon claramente en el bando contrario. Grotius, por ejemplo, conociendo a la perfección la obra de Victoria a quien cita habitualmente en sus textos, dejó dicho que "los mismos derechos (naturales) que tienen los hombres sobre su propiedad lo habilitan para convertirse en propiedad de otra persona, dado que es posible alienar una parte o una consecuencia de la propia libertad, y de este modo el otro adquiere un derecho sobre la misma, que se denomina derecho personal o jus in personam". Grotius, Hugo (1926) pp.295. 
efectivamente esos derechos inalienables de todos los miembros de la familia humana. A partir de la declaración del 48, los Estados miembros quedan comprometidos a asegurar -en cooperación con la organización de las Nacionales Unidas-, el respeto universal y efectivo a los derechos y libertades fundamentales del hombre (Considerando 6). Este compromiso es una obligación que nace de una relación fiduciaria entre los estados y los ciudadanos y autoriza a los ciudadanos a pedir cuentas a sus funcionarios cuando se constatan lesiones a los derechos humanos, porque los derechos humanos también son normas constitutivas del estado de derecho. Volveré sobre este punto al final.

Comprensiblemente no hay en la Declaración una trama conceptual diáfana que permita inferir de manera evidente el espíritu democrático republicano que animaba a algunos de sus redactores - no es casual que no la hubiera dado el pragmatismo triunfante que, quizás con razón, prefirió lograr una declaración aunque hubieran omisiones importantes-. Pero hay otros indicios -además de la inalienabilidad de la libertad- que podrían tomarse como ratio cognoscendi de tal espíritu republicano democrático. Por ejemplo, en el segundo considerando del Preámbulo asoma un texto claramente anti-hobbesiano: la libertad sólo sería concurrente con un mundo de seres humanos liberados del temor y la miseria. ${ }^{2}$ Nada más cercano a la concepción republicana de la libertad como no dominación arbitraria, incompatible con un diseño institucional que permita que unos vivan a merced de otros gracias al miedo que le infunden o a la miseria a la que los someten. Hobbes, en cambio, no se cansó de repetir que el miedo no es obstáculo sino el requisito para la realización de la libertad (personal y política), que el obstáculo no es el miedo sino la ley, que el inicio de la sociedad civil anida en el miedo mutuo, que la causa del miedo mutuo se debe tanto a la igualdad natural de los hombres como a la voluntad de hacerse daño mutuamente.

Los Preámbulos de 1789 y de 1793 guardan una clara analogía con el del 1948. El Preámbulo de 1789, por ejemplo, trazó una relación causal entre las calamidades públicas, la corrupción de los gobiernos y el desprecio de los derechos de los hombres; y la Declaración francesa de los Derechos del hombre y el Ciudadano, incluida en el Acta Constitucional de 1793, recuerda que el olvido y desprecio de los derechos naturales de los hombres son las únicas causas de las desgracias en el mundo. La Declaración de 1948 asumió un espíritu afín -adaptada a los nuevos acontecimientos y despojada del lenguaje iusnaturalista- y volvió a perfilar un nexo causal entre el menosprecio de los derechos humanos y los actos ultrajantes de barbarie, temor y miseria humanas. Algunos de los detractores de una y otra de las declaraciones -huelga decirlo- casi llegaron a expresar lo contrario. Así el caso del conservador y protoutilitarista Edmund Burke y del utilitarista Jeremy Bentham en el XVIII, ambos condenando los excesos teóricos y prácticos de la revolución francesa porque conducían al terror y la anarquía y, con algunas variaciones, la heideggeriana Arendt, vocera de la crítica burkeana y admiradora del pragmatismo del conservador inglés Burke, que hizo una crítica feroz de la declaración en nombre de un enigmático y famosísimo "derecho a tener derechos", que vendría a reemplazar la idealidad e hipocresía de los así llamados derechos de los hombres del 1948, en nombre de un impolítico derecho a la inclusión entendido como

2 Siete años antes, en 1941, en su "Discurso a la Nación”, Franklin Roosevelt había incorporado ya al miedo dentro de las causas de ilibertad al hablar las cuatro libertades: libertad de palabra, de expresión, de vivir sin penurias, de vivir sin miedo. 
un derecho a pertenecer a una comunidad organizada y como garantía de un lugar en el mundo que haga significativas sus opiniones y efectivas sus acciones. No hará falta recordar que Arendt reafirmó su famosa consigna recordando una y otra vez que se trataba de "una irónica, amarga y tardía confirmación de los famosos argumentos con los que Edmund Burke se opuso a la Declaración de Derechos del Hombre." (Arendt, 1949)³.

Los temores y las miserias están históricamente indexados, claro está, y en el 48 se trataba de terminar con una política mundial fundada en el nacionalismo, el colonialismo y racismo, perpetradora de masacres que dieron origen al término genocidio. En ese momento una parte de los miembros de la familia humana fueron despojados de su personalidad jurídica, sin derecho a una nacionalidad, a circular libremente, a participar en el gobierno de su país, a expresar sus opiniones, entre otras cosas. Restituir (al menos tendencialmente) la personalidad jurídica de todos los seres humanos -en todas partes- y en función de unos derechos inalienables e iguales de todos - a pesar de las omisiones, negociaciones y olvidos en la teoría y en la práctica- hizo renacer la teoría y praxis de los derechos humanos que -en el siglo XIX- habían enterrado los imperialismos y nacionalismos en la práctica y el utilitarismo y el positivismo jurídicos en la teoría.

\section{Los derechos inalienables deben ser universalizados a todos}

El primer considerando del "Preámbulo" de 1948 dice -un decir que estuvo a punto de hacer fracasar la declaración por las marcadas diferencias político filosóficas entre sus miembros- que: "la libertad, la justicia y la paz en el mundo tienen por base el reconocimiento de la dignidad intrínseca de los derechos iguales e inalienables de todos los miembros de la familia humana"; el artículo 3), por su parte, aclara que "todo individuo tiene derecho a la vida, a la libertad y a la seguridad de su persona."4

Los objetivos declarados -la libertad, la paz y la justicia en el mundo- se asientan sobre la intrínseca dignidad de los derechos inalienables (e iguales) de todos (los miembros de la familia humana). Nótese la doble referencia a conceptos normativos de carácter rotundamente universalista: se aspira a lograr justicia, paz y libertad en el mundo porque así lo requieren los derechos inalienables de todos los miembros de la familia humana.

No debemos caer en el error de ver en la frase "familia humana" un refuerzo normativo inicial para un concepto de familia que luego introduce uno de los articulados -gracias a una victoria lograda por los miembros más conservadores de la comisión- en el apartado 3) del artículo 16: "la familia es el elemento natural y fundamental de la sociedad". Se trata de una confusión fatal que opaca el valor de esa metáfora conceptual de la familia humana entendida como la unión fraternal (y por eso mismo horizontal) entre los seres humanos iguales en derechos y en dignidad (artículo 1); metáfora que cobra fuerza, también, al hablar de seres humanos que en todas partes tienen derecho al reconocimiento de su personalidad

3 Existe un buen comentario - que incluye una parte del texto de Burke- en Waldron, Jeremy (1987).

4 El libro de Glendon, M.A (2001), recoge una parte de las discusiones entre unos y otros en torno a la conveniencia o inconveniencia de introducir en el Preámbulo "un concepto de dignidad teórico, filosófico, metafísico e inconveniente, como quedó plasmado en los considerandos. Recomiendo también Hoffmann, Stefan Ludwig (Ed.). (2011), y el texto de Hunt, Lynn (2010). 
jurídica (artículo 6). ${ }^{5}$ Afirmar que en todas partes los seres humanos tienen derecho al reconocimiento de su personalidad jurídica -a realizar negocios jurídicos y contraer derechos y obligaciones adquiridas por sí mismos y sin depender de otros-, implica conferir a la humanidad en su conjunto (al menos tendencialmente) el carácter de sui iuris, de sujetos de derechos o, lo que es lo mismo, de sujetos libres republicanamente.

René Cassin -el jurista francés que devino una de las figuras intelectuales de la declaración- fue el redactor de uno de los tantos borradores que planteaba ligar el concepto de dignidad con el reconocimiento de la unidad de la humanidad y la garantía de la personalidad jurídica de todos, obviamente entendiendo que se trataba de confrontar las distintas ideologías nacionalistas y racistas:

"lo fundamental es que hay una unidad en la sociedad humana, sociedad compuesta por seres humanos que pueden compararse entre si, que tienen las mismas aptitudes naturales donde sea que estén, esto es lo más importante que debemos introducir en nuestra declaración" (...)”. Y continuaba diciendo que: "la idea de deberes recíprocos es el fundamento del concepto de fraternidad" (UN Commission on Human Rights $1947 \mathrm{e}){ }^{6}$

Cinco siglos antes, Bartolomé de las Casas ya lo había dicho en una grandiosa y sencilla frase: "la humanidad es una", espantado ante el genocidio perpetrado por España en América. Lo cierto es que en el Preámbulo de la Declaración cobra nueva vida la fraternidad revolucionaria francesa, acompañada por una idea republicana de una reciprocidad de deberes (y por tanto derechos) a escala global. Los deberes y derechos que le corresponden a la humanidad, considerada con una familia cuyos miembros deberían ser -todos ellospersonas jurídicas o sujetos por derecho propio (sui iuris).

\section{Libertad, igualdad y fraternidad}

Ni duda cabe que una de las mejores vías para interpretar un escrito es acercarse a las discusiones (del texto) en su propio contexto histórico; a los borradores, anotaciones y enmiendas, a las cartas de unos y otros, a la biografías de autores y críticos. Por eso son tan importantes $-\mathrm{y}$ fascinantes- los trabajos que cuentan en detalle las discusiones, borradores y

5 Obviamente hubo intensas discusiones en las reuniones de la Comisión de Derechos relativas a la conveniencia o inconveniencia de incluir el término "familia humana" en la Declaración. Fue René Cassin (el jurista francés, luego funcionario gaullista y Premio Nobel de la Paz) quien imprimió el sello del universalismo a las discusiones, apoyado por varios miembros de países no europeos que, entre otras cosas, vieron el uso del término como una herramienta crítica explícita ante el colonialismo, como cinco siglos antes ocurrió en la Escuela de Salamanca. Curiosa ironía fue que los representantes de estos pueblos fueran los defensores del cosmopolitismo, historia ésta que todavía no ha sido escrita en su totalidad. Sobre estas discusiones recomiendo el texto de Sluga, Glenda (2011).

6 El texto de Hoover es interesante, político y muy informativo; aunque incurre en algunos prejuicios metodológicos y en una falsa equiparación entre los derechos subjetivos y el liberalismo, que impiden al autor hacer uso de algunas de sus ideas interesantes y de matices importantísimos entre derechos alienables e inalienables. Un libro también instructivo, que recoge documentos y textos poco conocidos e importantes sobre derechos humanos es: Ishay, Micheline (2008). Especialmente importante para el tema que nos ocupa, es el capítulo 8, consagrado a analizar las discusiones sobre el Preámbulo y el artículo 1. 
enmiendas de las Declaraciones de Derechos. Afortunadamente hay varios libros de los buenos que narran historias de esos años, de los personajes en la escena, de los países o incluso continentes a los que representaban, ${ }^{7}$ contándonos, entre otras cosas, las idas y vueltas en torno al artículo 1 de la Declaración, que fue el último en recibir redacción definitiva: "todos los seres humanos nacen libres e iguales en dignidad y derechos y, dotados como están de razón y conciencia, deben comportarse fraternalmente los unos con los otros". Todos y cada uno de los conceptos que aparecen en el artículo -y en los demás- fueron discutidos una y otra vez y el resultado fue, obviamente, fruto de un consenso muchas veces forzado y de otras muchas de largas y fructíferas deliberaciones (y no de meras negociaciones). Ninguna crítica honesta de la redacción final debería pasar por alto los detalles de este proceso de elaboración, sin olvidar que las circunstancias en las que se discutía fueron producto creado por guerras imperialistas cuyas consecuencias siguen hoy azotando a la familia humana en su conjunto, y especialmente a sus miembros más débiles.

Prima facie, el artículo 1) dice que todos los miembros de la familia humana tienen dignidad intrínseca porque han nacido con derechos iguales e inalienables. ${ }^{8}$ Pero esta explicación es expedita pues omite dos conceptos muy discutidos (aunque no centrales) para el tema que nos ocupa: i) que los seres humanos nacen libres e iguales en dignidad y derechos y que ii) porque están dotados de razón y conciencia deben comportarse fraternalmente los unos con los otros. Digámoslo, aunque sea de pasada: i) que la inalienabilidad de los derechos le corresponda a los miembros de la familia humana por nacimiento no fue, obviamente un texto apreciado por los delegados de los países católicos decididos a extender derechos y dignidades hasta el momento mismo de la concepción, pero la querella se decantó por voto mayoritario a favor del nacimiento y ii) que los miembros de la familia humana, "dotados como están de razón y conciencia" deban comportarse fraternalmente los unos con los otros, fue una redacción poco feliz y ambigua, tanto si la razón y la conciencia designan características esenciales de los seres humanos, como si señalan los atributos necesarios para pertenecer como miembro a la familia humana. Basta con recordar que los perpetradores del genocidio no se cansaron de predicar que no todos los hombres son miembros de la familia humana, y que era preciso evitar los "esencialismos" en un texto político de semejante envergadura.

Pero la frase final del tardío y fundamental artículo no es ni filosófica ni políticamente ambigua: declara de manera categórica que la fraternidad es un deber exigible de manera universal, recuperando así un significado olvidado a mediados del XIX. La fraternidad jacobina -dentro y fuera de Francia- pretendía el ingreso de la inmensa mayoría de alieni iuris a la vida civil y política. En ese momento, para decirlo con Marx, había un enemigo común a vencer: los estamentos privilegiados del clero y la nobleza hereditaria, y en la lucha por la emancipación frente al enemigo cobró sentido la fraternidad como una forma de emancipación universal, un momento en que las aspiraciones y los derechos

7 es el capítulo 8, consagrado a analizar las discusiones sobre el Preámbulo y el artículo 1.

8 Sobre el concepto de dignidad como fundamento de los derechos humanos recomiendo el instructivo texto de Waldron, Jeremy (2013). Con el ánimo de echar luz sobre el carácter fundante de la dignidad, Waldron despeja varias dudas sobre el significado del término y, a pesar de las dificultades del término, hace votos por mantenerlo, recordando con Aristóteles, que no es inteligente exigir un grado de precisión no adecuado para conceptos como el dignidad porque 'se trata de cosas prácticas que siempre pueden ser de otra manera'. 
de la sociedad en su conjunto parecían coincidir con las aspiraciones y derechos de una determinada clase. El fracaso de los intentos revolucionarios por universalizar la libertad republicanamente entendida fue también el fracaso de la consigna fraternizadora francesa, que quedó relegada en el cajón de las reliquias de las que luego se ocuparía la religión o la moral de cada uno según su mayor o menor disposición o, en el mejor de los casos, permaneció agazapada en un sentimiento más o menos noble pero sin un anclaje institucional preciso ni precisable.

Cuando el liberalismo se encargó de alimentar la esperanza de mantener un orden social justo compatible con todo tipo de desigualdades materiales, el sentido de la fraternidad mudó en solidaridad e incluso en caridad cristiana, ambos ideales más ligados a la beneficencia que al reconocimiento universal de derechos.

Por eso no es de poca enjundia que en 1948 la Declaración de Derechos haya recuperado a la fraternidad como un deber positivo, como aquel que se nombra de manera muy general en el artículo 29): "toda persona tiene deberes respecto a la comunidad, puesto que sólo en ella puede desarrollar libre y plenamente su personalidad". Si el pleno desarrollo de la personalidad sólo es posible en comunidad, y si el pleno desarrollo de la personalidad implica derechos iguales para todos los miembros de la familia humana, el deber de la fraternidad no sería otra cosa que una lucha por el efectivo reconocimiento de los derechos iguales de todos los miembros de la familia humana. Las tres consignas revolucionarias francesas renacieron -aunque por un tiempo breve- hermanadas ante el enemigo común: el genocidio. Un nuevo contexto histórico y un terrible conjunto de circunstancias explican la utilidad de volver a invocarla.

Preciso es recordar, además, que en la Declaración de 1948 los derechos y deberes forman un todo sistemático, sin estériles y académicas distinciones entre derechos positivos y negativos o las libertades de los antiguos y los modernos. Todos ellos son derechos (positivos) derivados de un "concepto más amplio de libertad" del que habla el cuarto considerando; son derechos que requieren el esfuerzo de los pueblos y naciones a fin de asegurar su reconocimiento y aplicación efectiva a nivel nacional e internacional, tal como dice la Proclama de la Asamblea General.

Desde la perspectiva republicana del poder político, quizás podríamos interpretar la Proclama del siguiente modo: "los pueblos y naciones, las instituciones y los funcionarios públicos asumen el deber de asegurar el reconocimiento y aplicación de los derechos humanos -a nivel nacional e internacional- porque las instituciones, los gobiernos, los funcionarios públicos y los organismos internacionales detentan un poder sobre los individuos -fiduciariamente concedido- en nombre de los derechos de todos los miembros de la familia humana.

La interpretación fiduciaria del poder público autoriza a los estados (y organismos con poderes públicos delegados) a ejercer ese poder en nombre del pueblo y respetando la dignidad de los individuos en tanto sujetos libres y co-beneficiarios del poder de los estados. Eso implica al menos dos cosas:

i) si, como dejó dicho Locke, "todos los poderes... derivan del pueblo" entonces los funcionarios son sus trustees, y en todo momento tienen que rendir cuentas ante sus acciones públicas (o privadas) que lesionen los derechos o libertades de los beneficiarios y 
ii) en la interpretación fiduciaria de los poderes públicos, los derechos humanos son una parte constitutiva de la soberanía de los estados, una autorización para el uso del poder siempre que ese poder respete, proteja y haga efectivo el pleno reconocimiento de los derechos. Si los estados tienen que asegurar el imperio de la ley -la libertad igual de todos compatible con la de todos los demás-, y si los derechos humanos constituyen el suelo nutricio de ese imperio de la ley, entones los estados tienen el deber de gobernar internamente -y representar a sus ciudadanos internacionalmenteen función de un poder fiduciariamente concedido. (Criddle, 2009)

\section{Conclusión}

Una buena parte del olvido de la tradición democrática republicana histórica, y especialmente del carácter emancipatorio del valor político de la fraternidad, ha sido producto del triunfo del liberalismo en la primera parte del siglo XIX. Entre otras cosas, porque sus parientes cercanas y compañeras de lucha, la libertad y la igualdad, ya no parecían estar necesitadas de su compañía luego de haber sido depreciadas mediante intentos relativamente exitosos por convertirlas en premisas básicas de una concepción liberal de democracia que cristalizó en el primer tercio del XIX, y que es compatible con todo tipo de subordinación civil. El liberalismo decimonónico logró depotenciar el carácter revolucionario de la tríada, a fuerza de pergeñar un concepto de libertad -la desafortunadamente famosa libertad negativa de los modernos de Benjamin Constant y de Isaiah Berlin- entendida como ausencia de interferencia (real y actual pero no disposicional) compatible con todo tipo de dominación arbitraria. El olvido se debe, también, a que esta tradición escindió el potente vínculo trazado por el republicanismo histórico entre libertad e igualdad -en esta tradición, la igualdad no competía con la libertad sino que era, justamente la reciprocidad en la libertad, e impuso un modo de entender a la igualdad como un anodino concepto de "igualdad de oportunidades" o " de carreras abiertas" para todos, y olvidando así de manera consciente que ambas- la libertad y por esa misma razón también la igualdad- son incompatibles con la dependencia civil o política que soportan quienes carecen de todo tipo de propiedad personal. De esa manera se perdía o, mejor, se la apropiaron luego haciendo un uso discrecional del concepto los ultra liberales como R. Nozick, la poderosa idea propiedad de sí del republicanismo antiguo que significa -ni más ni menos- que ser un sujeto por derecho propio (sui iuris) o, lo que viene a ser lo mismo, gozar de la "capacidad de poder vivir sin tener que pedir permiso a otros."

De la mano de Rawls, el liberalismo anglosajón de izquierda le otorgó un nuevo significado a la fraternidad, como complemento y soporte del Principio de la Diferencia, o sea como normativamente ligada a la igualdad. Para Rawls la fraternidad es "la idea de que no querer tener mayores ventajas a menos que eso sea en beneficio de quienes están peor situados" e incluso "es la disposición personal de no aceptar recompensas que no promuevan el interés de todos". La consigna reapareció, pero el sentido de la fraternidad recuperado -al menos en la teoría- en la Declaración de 1948 ya se había perdido nuevamente cuando Rawls recordaba la divisa revolucionaria, relegada a auxiliar a una teoría de justicia distributiva no claramente compatible con otras disposiciones personales que no sean el autointerés, como ya hace tiempo lo advirtió Gerald Cohen. 


\section{Bibliografía}

Arendt, Hannah (2009). "Es gibt nur ein menschiges Recht” en http://www.hannaharendt. net/index.php/han/article/view/154/274. Consultado en mayo de 2018.

Burke, Edmund y Paine, Thomas (1961). Reflections on the Revolution in France and The Rights of Man: Dolphin, USA.

Fox Decent, E-Criddle, E (2009). “The fiduciary constitution of human rights”, Legal Theory 15: 301-336.

Glendon, M.A (2001). A World Made New. Eleanor Roosvelt and the Universal Declaration of Human Rights.

Grotius, Hugo (1977). The Jurisprudence of Holland, Scientia Verlag, Amsterdam.

Hoover, Joe, (2013). "Re-reading the universal declaration of human rights: Plurality, contestation, not consensus", Human Rights, 12(2), 217-241.

Hunt, Lynn (2010). Inventing Human Rights (2009), versión castellana de Beltran Ferrer, Jordi (2009) La invención de los derechos humanos, Tusquets, Buenos Aires.

Ishay, Micheline, 2008. The History of Human Rights: From Ancient Times to the Globalization Era. Berkley and Los Angeles, CA: University of California Press.

Kant, I. (1989). Metafísica de las Costumbres, Versión castellana de Cortina, Adela y Conill, Jesus: Tecnos, Madrid.

Locke, John (1998). Segundo Tratado sobre el gobierno civil, Alianza Editorial, Buenos Aires.

Morsink, Johannes (1999). The Universal Declaration of Human Rights: Origins, Drafting and Intent, University of Pennsylvania Press, USA.

Sluga, Glenda (2011). "Renee Cassin Les droits de l'homme and the Universality of Human Rights, 1945-1966" en Hoffmann, Ludwig, Stefan (ed.) Human Rights Human Rights in the Twentieth Century , Cambridge, Cambridge U.P.

UN Comission of Human Rights (1947e). "Verbatim Record, 4 February 1947”: Charles Malik Papers, Library of Congress, Manuscripts Division.

Waldron, Jeremy (Ed.) (1987). Nonsense upon Stilst: Bentham, Burke and Marx on the Rights of Man, Methuen, London.

Waldron, Jeremy (2013). “¿Is Dignity the foundation of Human Rights?”, New York University Public Law and Legal Theory, Working Papers, Paper 374, New York. 
